\title{
Contribuições da Atividade física no envelhecimento dos idosos
}

\author{
Contributions of physical activity in the aging of the elderly \\ Contribuciones de la actividad física en el envejecimiento de las personas mayores
}

Recebido: 27/12/2020 | Revisado: 28/12/2020 | Aceito: 31/12/2020 | Publicado: 02/01/2021

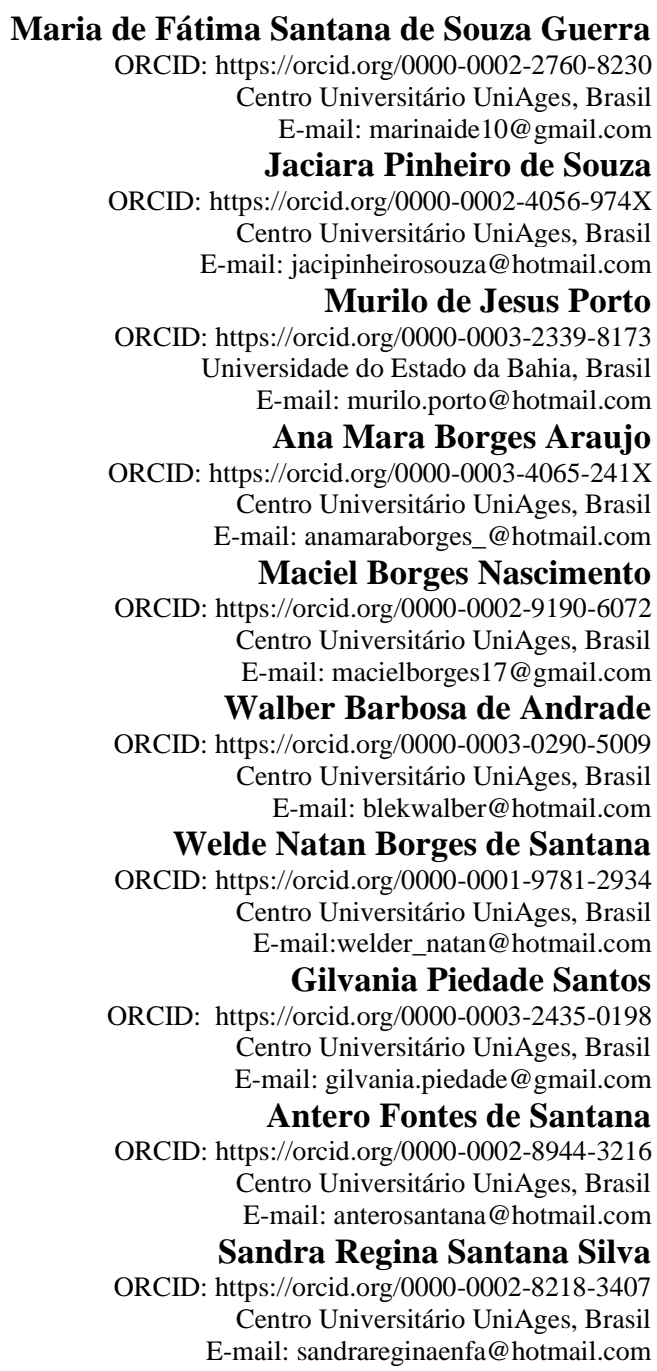

\begin{abstract}
Resumo
A característica do envelhecimento não depende apenas da condição genética, mas sobretudo, do estilo de vida. Portanto, para entender a etiologia associada aos processos degenerativos que lhe estão associados, é fundamental conhecer e desenvolver estratégias que atenuem os efeitos da senescência e da senilidade. Logo, o estudo tem como objetivo analisar a influência da atividade física no aspecto social dos idosos através de um levantamento bibliográfica. A metodologia usada foi a revisão narrativa, utilizando bases de dados online como sites confiáveis; revistas eletrônicas; livros; jornais. O levantamento de dados não se pautou para restrição do ano de publicação dos trabalhos encontrados. Na busca de dados, foram escolhidos os trabalhos que contemplasse o tema em questão, valendo-se de uma leitura prévia dos títulos; resumos ou prólogo ou texto introdutório a depender do tipo de publicação; por fim a leitura do texto por inteiro. Os resultados revelam que a inserção de um estilo de vida ativo, através da prática de atividade física, proporciona benefícios biopsicossocial e espiritual à saúde do idoso, uma vez que é considerado como um importante componente para a melhoria da qualidade de vida e da sua independência funcional. Destarte, a partir desta revisão de literatura, conclui-se que o método de atividade física contribui positivamente para o envelhecimento saudável do idoso e que, decorrente do aumento da longevidade e do decréscimo progressivo da taxa de natalidade, ocorrerá o aumento dessa população nos próximos anos.
\end{abstract}

Palavras-chave: Envelhecimento; Idoso; Atividade física. 


\begin{abstract}
The characteristic of aging does not depend only on the genetic condition, but above all, on the lifestyle. Therefore, in order to understand the etiology associated with the degenerative processes associated with it, it is essential to know and develop strategies that mitigate the effects of senescence and senility. Therefore, the study aims to analyze the influence of physical activity on the social aspect of the elderly through a bibliographic survey. The methodology used was narrative review, using online databases as reliable sites; electronic magazines; books; newspapers. The data collection was not restricted to the year of publication of the works found. In the search for data, the works that contemplated the theme in question were chosen, making use of a previous reading of the titles; abstracts or prologue or introductory text depending on the type of publication; finally the reading of the entire text. The results reveal that the insertion of an active lifestyle, through the practice of physical activity, provides biopsychosocial and spiritual benefits to the health of the elderly, since it is considered as an important component to improve the quality of life and their independence functional. Thus, from this literature review, it is concluded that the physical activity method contributes positively to the healthy aging of the elderly and that, due to the increase in longevity and the progressive decrease in the birth rate, this population will increase in the coming years.
\end{abstract}

Keywords: Aging; Old man; Physical activity.

\title{
Resumen
}

La característica del envejecimiento no depende solo de la condición genética, sino sobre todo, del estilo de vida. Por tanto, para comprender la etiología asociada a los procesos degenerativos asociados a ella, es fundamental conocer y desarrollar estrategias que mitiguen los efectos de la senescencia y la senilidad. Por tanto, el estudio tiene como objetivo analizar la influencia de la actividad física en el aspecto social de las personas mayores a través de una encuesta bibliográfica. La metodología utilizada fue la revisión narrativa, utilizando bases de datos en línea como sitios confiables; revistas electrónicas; libros; periódicos. La recogida de datos no se limitó al año de publicación de los trabajos encontrados. En la búsqueda de datos se eligieron las obras que contemplaban el tema en cuestión, utilizando una lectura previa de los títulos; resúmenes o prólogo o texto introductorio según el tipo de publicación; finalmente la lectura de todo el texto. Los resultados revelan que la inserción de un estilo de vida activo, a través de la práctica de actividad física, brinda beneficios biopsicosociales y espirituales a la salud de las personas mayores, ya que se considera un componente importante para mejorar la calidad de vida y su independencia. funcional. Así, de esta revisión de la literatura se concluye que el método de actividad física contribuye positivamente al envejecimiento saludable de las personas mayores y que, debido al aumento de la longevidad y al descenso progresivo de la tasa de natalidad, esta población se incrementará en los próximos años.

Palabras clave: Envejecimiento; Anciano; Actividad fisica.

\section{Introdução}

O envelhecimento é um processo fisiológico do desenvolvimento humano responsável por desencadear uma série de transformações no estilo de vida da pessoa idosa. De modo que é notória a propensão do desencadeamento das doenças crônicas, bem como o desenvolvimento de alterações musculoesqueléticas que surgem nesta fase, as quais são determinadas por uma série de alterações metabólicas e endócrinas que vão surgindo de acordo com o envelhecer (Freitas, 2006).

Até o século passado, as principais doenças que diminuíam a longevidade eram as infecciosas, agravadas pelas deficiências nutricionais, de saneamento e de transporte. Entretanto, essas foram reduzidas a partir da inserção de políticas públicas tais como a medicina preventiva, as vacinas e os investimentos na educação popular. Porém nos dias atuais, as enfermidades fatais são, frequentemente, causadas pelo consumo excessivo de calorias, gordura, álcool, tabagismo, etilismo e o sedentarismo os quais, em longo prazo, desencadeiam doenças crônicas (Freitas, 2006).

Vale ressaltar que atrelado à longevidade houve um aumento das doenças crônicas, de modo que segundo os Princípios do Sistema Único e Saúde - SUS se faz necessário qualificar os profissionais de saúde para o atendimento a essa demanda com necessidades diferenciadas (Brasil, 2011). É necessário que se tenha uma visão holística e mais atualizada sobre o cuidado destinado a esse público, para que se tenha um atendimento integral e resolutivo.

Tendo em vista tais preceitos, pesquisadores demonstram a importância da atividade física no sentido de melhorar a qualidade de vida destes idosos, implantando assim projeto de atividades físicas já que estudos demonstram que as pessoas ativas apresentam menor incidência de desenvolver doenças crônicas degenerativas - D.C.D (Madeira et al. 2013). 
Mediante contextualização, é notório o aumento do número de idosos mundialmente e a ainda a pouca adesão dos idosos à atividade física, fazendo surgir a inquietação acerca do tema e o pensamento crítico para conhecer através de metodologia específica a realidade deste público. Nesse sentido o objetivo desse trabalho foi analisar a influência da atividade física no aspecto social dos idosos através de um levantamento bibliográfico.

\section{Metodologia}

Este estudo trata-se de uma revisão narrativa, com abordagem exploratória qualitativa a nível bibliográfico. Desta forma analisa-se os presentes dados da literatura teórica com as devidas pesquisas, além de agrupar vasto leque de propósitos: conceito sobre a temática discutida, analise de referenciais teóricos, e avaliação de problemas metodológicos.

A pesquisa de caráter bibliográfica qualitativa é a possibilidade de averiguar e abranger, por meio de dados estatísticos de determinados fenômenos, a intuição e a subjetividade, tendo como análise primordial a exposição das características de uma determinada população e também o aperfeiçoamento de ideias e a classificação de informações necessárias para a realização da pesquisa (Gil, 2010).

Inicialmente, foi realizado a busca de produções que albergasse a importância da atividade física para a saúde do idoso e por fim como ocorre o seu processo de envelhecimento, tendo como objetivo analisar a influência da atividade física no aspecto social dos idosos.

Na busca de dados, foram escolhidos os trabalhos que contemplasse o tema em questão, valendo-se de uma leitura prévia dos títulos; resumos ou prólogo ou texto introdutório a depender do tipo de publicação; por fim a leitura do texto por inteiro.

O processo metodológico abordado para a coleta foi a realização de um estudo, através de um levantamento bibliográfico por meio da busca eletrônica no Scielo, Lilacs e Google Acadêmico, a partir de palavras - chaves: Envelhecimento saudável; atividade física no idoso; senilidade. Assim, foram utilizados como critérios de inclusão: veículo de publicação: artigos, dissertações, teses, livros e manuais; idioma de publicação: português. Utilizou-se como critério de exclusão: artigos de outra temática; revisão sistemática, metanálise, editoriais e relatos de casos.

\section{Resultados e Discussão}

\section{Processo Fisiológico do Envelhecimento}

A característica do envelhecimento pode variar de um indivíduo para o outro, e está diretamente relacionado aos fatores biológicos, psicológicos, condições socioeconômicas, estilo de vida, dentre outros fatores que têm influência direta nesse processo. Portanto, se reportar ao envelhecimento é discutir um leque de interpretações variadas que se enlaçam ao habitual e a perspectivas de culturas distintas (Fechine \& Trompieri, 2012).

O envelhecimento normal atinge todos os humanos, pois é algo geneticamente normal para nossa espécie e independe dos fatores ambientais e patológicos. São diversas as alterações fisiológicas que acontecem em nosso corpo na velhice, desde estruturais a funcionais que podem ser agravados a partir do estilo de vida do idoso desde a sua juventude (Fechine \& Trompieri, 2012).

A linha que diferencia senescência de senilidade é muito estreita, as modificações fisiológicas do envelhecimento não devem ser tratadas como doenças. Os idosos saudáveis tem uma menor reserva funcional de seus órgãos e sistemas, com manutenção das funções orgânicas. Sendo assim, é importante destacar que existem as mudanças normais e patológicas que caracterizam o envelhecimento senescente e senil (Costa et al., 2005).

Dentre as modificações fisiológicas gerais estão: a diminuição da estatura, a partir de 40 anos perde-se $1 \mathrm{~cm}$ a cada década; o aumento da curvatura da coluna vertebral; a diminuição do arco dos pés; o achatamento dos discos; o tecido celular 
adiposo diminui nos membros e aumenta no tronco e órgãos; há o aumento do diâmetro da caixa torácica; redução da massa muscular e da elasticidade da pele, que torna-se mais áspera, flácida e ressecada; branqueamento e afinamento dos cabelos e calvície, dentre outras modificações comuns (Costa et al., 2005).

As mudanças também ocorrem com os sentidos. Na audição ocorre a calcificação das articulações entre os ossículos e uma consequente diminuição da audição e o ressecamento e aumento de cera. Na visão, a capacidade de acomodação do cristalino diminui, o olho torna-se mais sensível à luminosidade, há perda de nitidez das cores, o cristalino passa por um processo de opacificação, a catarata; e é comum a redução do campo visual. No olfato os receptores olfativos diminuem, assim como a capacidade olfativa de identificar o tipo e a intensidade do cheiro. No paladar os botões gustativos atrofiam, diminuindo o paladar, principalmente para salgado e com o processo de envelhecimento o limiar gustativo aumenta (Costa et al., 2005).

A nível cardiovascular, ocorrem alterações em marcadores biológicos esperadas com o tempo de funcionalidade celular do idoso, que quando submetido a determinados esforço de caráter físico, resultando em constante queda na aptidão do cardio de elevar o número e a força dos batimentos cardíacos em geral, assim outro fator a ser respondido, mostra também a redução da frequência cardíaca em repouso, elevação do colesterol e da resistência vascular periférica, o que geral como produto final a elevada tensão arterial. Com o processo do envelhecimento, a frequência cardíaca não sofre qualquer modificação relevante, em repouso. Já na frequência cardíaca máxima em exercício, resulta em um declínio bastante significativo (Fechine \& Trompieri, 2012).

Essas alterações relacionadas à idade acabam diminuindo a eficácia do coração e colaboram para diminuição da resistência do musculo cardíaco. As paredes das veias e artérias ficam mais tortuosas devido ao acúmulo de gordura e cálcio em seus interiores (Brunner \& Suddarth, 2009).

A avaliação cuidadosa dos idosos é necessária porque eles frequentemente se apresentam com diferentes sintomas das pessoas mais jovens. As pessoas idosas são mais prováveis de apresentar dispneia ou sintomas relacionados à cardiopatia e podem experimentar alterações do estado mental ou relatar sintomas vagos como fadiga, náuseas e síncope. Em lugar da típica dor torácica subesternal associada à isquemia miocárdica, os pacientes idosos podem relatar a dor em queimação ou aguda ou desconforto em uma área da região superior do corpo (Brunner \& Suddarth, 2009).

Nesse mesmo contexto se evidencia o aumento de casos de arteriosclerose, desencadeada pelo acúmulo de gordura, o aumento de colágeno e a perda de fibras elásticas. Dessa forma, as funções cardiovasculares acabam prejudicadas, o que traz complicações ao idoso, dentre elas destaca-se a diminuição do tempo resposta de elevação cardíaca ao esforço ou algum estímulo. Por isso há uma maior prevalência nos casos de hipertensão arterial sistólica, o que aumenta as chances de acidentes cardiovasculares (Silverthon, 2010).

Durante o processo de envelhecimento incide a diminuição da função pulmonar. Para os homens, a redução dessa função é o principal fator de risco para incidência de doença coronária. A função pulmonar aumenta durante a adolescência, estabiliza até o período dos 30 anos e, depois disso, começa a declinar. Algumas alterações estruturais no aparelho respiratório são evidentes com o envelhecimento, a caixa torácica se torna mais rígida, ocorre à diminuição da elasticidade pulmonar e das ventilações (Fechine \& Trompieri, 2012).

As alterações fisiológicas na senescência no pulmão do idoso podem ser ocasionadas pelas combinações entre alterações anatômicas e as reorientações das fibras elásticas. Essas alterações fisiológicas são definidas pela diminuição da elasticidade pulmonar, redução da capacidade da difusão do oxigênio, redução dos fluxos expiratórios, elevação da complacência pulmonar, fecho das pequenas vias aéreas e fecho prematuro de vias aéreas (Fechine \& Trompieri, 2012).

As principais alterações funcionais do aparelho respiratório durante o processo de envelhecimento são a redução da complacência da parede torácica e da força dos músculos respiratórios; além da diminuição capacidade vital, da pressão 
arterial de oxigênio, da taxa de fluxo expiratório, da difusão pulmonar de $\mathrm{CO} 2$, da sensibilidade respiratória à hipóxia; aumentam os volumes residuais, mas mantêm a capacidade pulmonar total (Fechine \& Trompieri, 2012).

Embora os sintomas gastrintestinais causem menos riscos que os problemas cardiovasculares ou respiratórios, eles podem causar incômodo e preocupar mais as pessoas idosas, isso porque esse sistema é alterado em todos os aspectos pelo processo de envelhecimento. As mudanças vão desde os dentes na boca até estruturas acessórias, como o fígado, que afetam a função gastrintestinal (Eliopoulos, 2011).

O envelhecimento também torna evidentes as modificações no sistema musculoesquelético com a diminuição do crescimento, elasticidade e do número de fibras. Há perda de massa muscular e da elasticidade dos tendões e ligamentos além da viscosidade dos fluidos sinoviais também é notável (Fechine \& Trompieri, 2012). A massa muscular assim como os movimentos e a força ficam diminuídos, essas mudanças são evidentes nos músculos dos braços e pernas, que tornam-se mais fracos e flácidos (Eliopoulos, 2011).

Pelo fato de as variações na frequência dessas mudanças serem capazes de sugerir que resultam mais da inatividade que do envelhecimento, a importância do exercício para minimizar perda de tônus e força muscular deve ser sempre enfatizada. Os tendões encolhem e enrijecem, causando redução nos reflexos profundos. Os reflexos diminuem nos braços, sendo que quase que completamente perdidos no abdome, mas não são mantidos nos joelhos. Por muitas razões, cãibras musculares ocorrem com frequência (Eliopoulos, 2011).

No sistema urinário, ocorre à diminuição do tamanho dos rins, do número de glomérulos, do fluxo sanguíneo renal e da capacidade de concentração e diluição urinárias. Há também uma menor eficiência dos mecanismos compensatórios e um aumento do tecido fibroso que substitui os glomérulos e a distribuição de hidroeletrolíticos e ácidos básicos têm uma menor adaptação. Dos 30 aos 70 anos, ocorre o declínio de 50\% da reserva funcional (Costa et al., 2005).

O Sistema Nervoso Central (SNC) é o sistema biológico mais comprometido com o envelhecimento. Ele é responsável pelas sensações, movimentos, funções psíquicas e pelas funções biológicas internas (Fechine \& Trompieri, 2012).

Segundo Cançado e Horta (2002), o que preocupa no envelhecimento é o fato de o SNC não possuir capacidade reparadora. Esses autores expressam que o SNC é definido como unidades morfofuncionais pós-mitóticas, sendo estas sem possibilidades reprodutoras, estando sujeito ao envelhecimento decorrente de fatores intrínsecos (genética, sexo, sistema circulatório e metabólico, radicais livres, etc.) e extrínsecos (ambiente, sedentarismo, tabagismo, drogas, radiações, etc) (Fechine \& Trompieri, 2012).

Com o passar dos anos ocorrem muitas mudanças no sistema reprodutivo feminino, a vulva atrofia por causa de mudanças hormonais, há perda de gordura subcutânea e de pelos e o achatamento dos lábios. Nas mulheres idosas a região vaginal é mais alcalina, sua flora muda e suas secreções reduzem; a vagina tem aparência rosada e ressecada além da presença de rugas. Nos homens, à medida que envelhecem o epitélio afina e as vesículas seminais são afetadas pela suavização da mucosa, o tecido muscular é substituído pelo conjuntivo e sua capacidade de reter líquidos é reduzida e ocorre um pouco de atrofia dos testículos. Também é comum o aumento da próstata, e, diferente do que muitos pensam o idoso não perde a capacidade física para ter ereções ou ejaculações (Eliopoulos, 2011).

\section{A Influência da Atividade Física na Saúde do Idoso}

Assim, a população em geral transformou seu perfil, aparecendo uma nova realidade. $\mathrm{O}$ aumento quantitativo de idosos, somado à queda da natalidade, são circunstâncias em um país estimado jovem, mas com propriedades que nos organizam para uma população acima de 60 anos (Herédia, et al., 2007).

Conforme o Instituto Brasileiro de Geografia e Estatística, somente até 2030, o quantitativo de pessoas com mais de 60 anos de idade deverá superar o de crianças e adolescentes em cerca de 4 milhões, contestação que adicionará para 35,8 
milhões em 2050. Com o acréscimo da expectativa de vida, conforme a Organização Mundial da Saúde - OMS, a população brasileira estará no ranque de $6^{\circ}$ país do mundo com o maior número de pessoas idosas até 2025 (Ibge, 2009).

A característica em geral do envelhecimento pode ser bem-conceituado de distintas formas, dentre as quais a definição mais concreta, está voltado para perda da eficiência dos processos envolvidos na sustentação da homeostase do organismo, provocando no decréscimo de variável ou aumento de outras questões como o estresse. Dentro desse conjunto, as deformações morfológicas e funcionais voltadas para o envelhecimento compõem uma das maiores apreensões para os profissionais da área da saúde, principalmente no que se refere à cautela de doenças e desenvolvimento da qualidade de vida (Robergs, 2002).

Assim, a atividade física regular e sistematizada ou exercícios físicos aceitáveis têm evidenciado serem capazes de tornar menor as decorrências deletérios do envelhecimento, como a redução na massa muscular, contribuindo para a conservação da capacidade física e autonomia do idoso. Essencialmente, já é admissível observar um maior número de fluxogramas de exercícios físicos tornados a pessoa idosos. (Reeves, 2006)

Nesse contexto a atividade física, exercício físico e aptidão física são termos que definem diferentes conceitos, mas são frequentemente confundidos e usados para definir a mesma coisa. Atividade física pode ser definida como qualquer movimento corporal executado pelos músculos esqueléticos que resultam gasto energético que pode ser medido em quilocalorias. A atividade física, na vida diária, pode ser categorizada como: ocupacional, esportiva, condicionante, ocupações domésticas e outras atividades (Brasil, 2010).

O exercício físico é uma parte planejada e estruturada do conjunto de atividades físicas e é praticado de forma repetitiva tendo como objetivo, intermediário ou final, manter ou melhorar a aptidão física. Por outro lado, ao conjunto de atributos relacionados com a saúde e com o nível das habilidades associadas a tais atributos, denominamos aptidão física. O grau aptidão física apresentado pelas pessoas pode ser medido com testes específicos. Portanto fica claro que todos, de alguma forma, praticam atividades físicas, mas poucos fazem exercícios físicos para ganhar a aptidão física imprescindível para uma vida saudável (Brasil, 2010).

Entretanto, em virtude de a maioria da população não possuir acesso a programas de atividade física orientada, por questões financeiras ou ausência de interesse pessoal, torna-se importante manter os níveis de atividade física por meio das tarefas de vida diárias (caminhar, higiene pessoal, levantar sem auxílio), bem como atividades instrumentais da vida diária (utilizar meios de transporte, lavar sua roupa, fazer compras e administrar os próprios medicamentos). Sendo assim, manter as atividades de vida diária, como varrer a casa ou ir ao supermercado, também pode ser um recurso importante para minimizar os efeitos degenerativos provocados pelo envelhecimento, que são agravados com o sedentarismo (Acsm, 1999).

\section{Considerações Finais}

Mediante o levantamento bibliográfico, conclui-se que a prática de atividade física contribui positivamente para o envelhecimento saudável do idoso, sendo evidente que envelhecer com saúde é um processo fisiológico e natural e que, decorrente do aumento da longevidade e do decréscimo progressivo das taxas de natalidade, ocorrerá o aumento dessa população nos próximos anos.

É justamente a mudança desse perfil da pirâmide etária, que tem despertado a atenção da comunidade acadêmica em desenvolver pesquisas e, do Estado em elaborar políticas públicas direcionadas para o envelhecimento saudável. Assim sendo, se faz necessário entendê-lo como um processo fisiológico do desenvolvimento humano, ou seja, senescência. Nesse sentido, se faz importante à inserção do idoso como ser integral na sociedade, através de políticas públicas, grupos sociais e atividades preventivas, já que muitas doenças crônicas são advindas do seu estilo de vida. A prática de atividades físicas é um importante meio para se alcançar esses objetivos, devendo ser estimulada ao longo da vida, especificamente nessa faixa etária, possibilitando assim que esse ciclo de vida seja de uma forma autônoma e qualitativamente positiva. 
Esta revisão bibliográfica procurou fornecer subsídio literário sobre as diversas formas de como o adulto idoso envelhece, permitindo ou tentando permitir um maior conhecimento sobre o tema. Contudo o interesse dessa pesquisa é disponibilizar os conhecimentos teóricos para que possam ser utilizados no campo prático, vislumbrando maior acesso ao conhecimento que proporcionará melhor qualidade de vida dos idosos.

\section{Referências}

Acsm. (1999). Programa de condicionamento físico da ASM. (2a ed.). Manole.

Brasil. (2010). Envelhecimento e saúde da pessoa idosa. Série A. Normas e Manuais Técnicos Cadernos de atenção básica, 19.

Ministério da Saúde. (2006). Revista Brasileira Saúde da Família Ministério da Saúde Secretaria de Atenção à Saúde Departamento de Atenção Básica. Brasília.

Ministério da Saúde (2013). Conselho Nacional de Saúde. Resolução no 466, de 12 de dezembro de 2012. Publicada no DOU no 12 - quinta-feira, Seção 1 , p 59.

Ministério da Saúde (2014). Abc do SUS doutrinas e princípios. Brasília/DF. 2011. Disponível em: http://biblioteca.planejamento.gov.br/biblioteca-tematica$1 /$ textos/saude-epidemias-xcampanha.

Brunenr \& Suddar. (2009). Tratado de Enfermagem Médico- Cirúrgico. Rio de Janeiro: Guanabara Koogan.

Cançado, F. A. X., \& Horta, M. L. (2002). Envelhecimento cerebral In E. V. Freitas., L. Py., A. L. Néri., F. A. X. Cançado., M. L. Gorzoni, M. L., \& S. M. Rocha (Eds), Tratado de Geriatria e Gerontologia. Rio de Janeiro: Guanabara Koogan, 112-127.

Costa, E. F. A., \& Pereira, S. R. M. (2005). Meu corpo está mudando o que fazer? In J. L. Pacheco, J. L. M. Sá, L. Py, \& S. N. Goldman (Orgs.).

Fechine, B. R. A., \& Trompieri, N. (2012). O processo de envelhecimento: as principais alterações que acontecem com o idoso com o passar dos anos. InterscienceplacE, 1(20).

Eliopoulos, C. (2011). Enfermagem Gerontológica. (7a ed.), Porto Alegre: Artmed.

Freitas, G. F., \&Oguisso, T. (2010). Ética no Contexto da Prática de Enfermagem. Med. book Editora Científica. Rio de janeiro-RJ.

Gil, A. C. (2010). Como Elaborar Projetos De Pesquisa. (5a ed.), São Paulo: Atlas.

Herédia V., Ferla A., \&Lorenzi D. (2007). Envelhecimento, saúde e políticas públicas. Caxias do Sul- RS: Educs.

Ibge. (2000). Censo 2000. Rio de Janeiro: IBGE.www.tabnet.datasus.gov.br.

Ibge. (2008). Síntese de indicadores sociais: uma análise das condições de vida da população brasileira. IBGE. http://www.ibge.gov.br/ home/estatistica/populacao/condicaodevida/indicadoresminimos/sintese.

Ibge. (2009). Indicadores sociodemográficos e de saúde no Brasil. IBGE. http://www.ibge.gov.br/home/estatistica/populacao /condicaodevida/indicadoresminimos/sintese.

Madeira. M. C. (2013). Atividade física no deslocamento em adultos e idosos do Brasil: prevalências e fatores associados. Cad. Saúde Pública. 29(1).

Reeves, N. D., Narici, M. V., \& Maganaris, C. N. (2006). Myotendinous plasticity to ageing and resistance exercise in humans. Exp. Physiol; 9(3), 483-498.

Robergs, R. A. \& Roberts, S. O. (2002). Princípios fundamentais de fisiologia do exercício para aptidão, desempenho e saúde. Phorte.

Silverthorn, D. U. (2010). Fisiologia Humana, (5a ed.), Artmed.

Tannure, M. C. (2010). SAE: sistematização da assistência de enfermagem. Guanabara Koogan. 